\title{
Factores asociados a la infección y muerte por Covid-19, un análisis ecológico
}

\author{
Factors associated with infection and death from Covid-19, an ecological analysis \\ Fatores associados à infecção e morte de Covid-19, uma análise ecológica
}

\section{Franz Chumbi Sigcho}

fchumbisigcho@gmail.com

https://orcid.org/0000-0002-3247-4273

\section{Juan Moreno Bravo}

juanpamoreno001@gmail.com

https://orcid.org/0000-0002-9803-3788

\author{
Carlos Román Collazo \\ cromanc@ucacue.edu.ec \\ https://orcid.org/0000-0002-8235-4165
}

\author{
Jessica Velecela Chumbi \\ jessy2012_20@hotmail.com \\ https://orcid.org/0000-0003-0373-7326
}

\section{Programa de Maestría en Diagnóstico de Laboratorio Clínico y Molecular, Universidad Católica de Cuenca, Cuenca-Ecuador}

Recibido 28 de septiembre 2021 / Arbitrado y aceptado 19 de octubre 2021 / Publicado 30 de diciembre 2021

\begin{abstract}
RESUMEN
La pandemia del SARS-CoV-2 y Covid-19 ha sido una amenaza a la vida en todo el planeta. El objetivo del presente estudio fue identificar los factores de riesgo asociados a la infección y mortalidad por Covid-19 a nivel global. Se realizó un análisis ecológico de los factores asociados en 91 países pertenecientes los cinco continentes del planeta. Los datos fueron tomados de la base de datos de acceso libre creada por la organización Our World in Data adscrita a la Universidad de Oxford para tres fechas 30 de junio y 30 de noviembre del año 2020 así como, 4 de junio del 2021. Se analizaron 11 variables predictoras para la infección y muerte por Covid 19 mediante correlación y regresión lineal múltiple en el programa SPSS 25.0. Existió correlación de 7 variables; sin embargo, solo algunas tienen poder explicativo. Los modelos para las tres fechas fueron diferentes. La fracción de adultos mayores de 65 años o más fue la única variable que explica los contagios por país en las tres fechas estudiadas. Las muertes fueron explicadas por el IDH en el mes de junio del año 2020, la proporción de adultos mayores a 65 años en el mes de noviembre del año 2020 y junio del 2021. La fracción de mujeres fumadoras también fue explicativa en junio del. Los hallazgos investigativos corroboran y contradicen investigaciones previas mostrando la heterogeneidad y complejidad en la interacción de los determinantes en salud en el ámbito de la pandemia de Covid-19.
\end{abstract}

Palabras clave: Covid 19; Mortalidad; Infección; Edad; Tabaquismo en mujeres

\begin{abstract}
The SARS-CoV-2 and Covid-19 pandemic has been a threat to life across the planet. The objective of this study was to identify the risk factors associated with Covid-19 infection and mortality at a global level. An ecological analysis of the associated factors was carried out in 91 countries belonging to the five continents of the planet. The data were taken from the open access database created by the Our World in Data organization attached to the University of Oxford for three dates June 30 and November 30, 2020 as well as June 4, 2021. They were analyzed 11 predictor variables for Covid 19 infection and death by correlation and multiple linear regression in the SPSS 25.0 program. There was a correlation of 7 variables; however, only some have explanatory power. The models for the three dates were different. The fraction of adults older than 65 years or more was the only variable that explains the infections by country in the three dates studied. The deaths were explained by the HDI in June 2020, the proportion of adults over 65 years in the month of November 2020 and June 2021 . The fraction of women smokers was also explanatory in June of. The research findings corroborate and contradict previous research showing the heterogeneity and complexity in the interaction of health determinants in the field of the Covid-19 pandemic.
\end{abstract}

Key words: Covid 19; Mortality; Infection; Age; Woman moking 


\section{RESUMO}

A pandemia SARS-CoV-2 e Covid-19 tem sido uma ameaça à vida em todo o planeta. O objetivo deste estudo foi identificar os fatores de risco associados à infecção e mortalidade por Covid-19 em nível global. Foi realizada uma análise ecológica dos fatores associados em 91 países pertencentes aos cinco continentes do planeta. Os dados foram retirados do banco de dados de acesso aberto criado pela organização Our World in Data anexada à University of Oxford para três datas 30 de junho e 30 de novembro de 2020, bem como 4 de junho de 2021. Foram analisadas 11 variáveis preditoras para Covid 19 infecção e morte por correlação e regressão linear múltipla no programa SPSS 25.0. Houve uma correlação de 7 variáveis; no entanto, apenas alguns têm poder explicativo. Os modelos para as três datas eram diferentes. A fração de adultos com mais de 65 anos foi a única variável que explica as infecções por país nas três datas estudadas. As mortes foram explicadas pelo IDH em junho de 2020, proporção de adultos com mais de 65 anos no mês de novembro de 2020 e junho de 2021 . A fração de mulheres fumantes também foi explicativa em junho de. Os resultados da pesquisa corroboram e contradizem pesquisas anteriores, mostrando a heterogeneidade e complexidade na interação dos determinantes da saúde no campo da pandemia de Covid-19.

Palavras-chave: Covid 19; mortalidade; infecção; era; fumar em mulheres

\section{INTRODUCCIÓN}

La pandemia de Covid-19, con su impacto epidémico, sanitario, económico y social ha situado a las enfermedades infecciosas emergentes a la cima de la lista de desafíos mundiales. Reportes iniciales de la pandemia en el mes de febrero del 2020 mostraron un total de 82623 casos y 2858 muertes. El crecimiento de infectados y muertes ha sido tal que en la actualidad sobrepasan los millones de personas (1).

Los datos de los CDC revelaron una tasa de muerte del $0.25 \%$ al $3 \%$. Las estimaciones varían según la ubicación de la enfermedad: oscilan entre el $1,17 \%$ en Corea del Sur, el $2,3 \%$ en China 25 y el 7,2\% en Italia 2,6. Las tasas de mortalidad pueden ser más altas en los epicentros de la enfermedad debido a un fallo sistémico o reducción de recursos para los programas de salud, lo que conduce al aumento de la mortalidad (2).

Las estimaciones de mortalidad también pueden verse influidas por diferentes variables como la demografía de la población, las diferencias en los programas de detección, estado de salud entre otros(3). Como otras enfermedades infecciosas, el Covid-19 muestra un cuadro clínico muy variable, que va desde asintomático hasta la muerte. En países europeos, los hombres mayores, con una o más comorbilidades, han sido el grupo más sensible con mayor mortalidad y morbilidad, incluido el síndrome de dificultad respiratoria fatal como complicación más frecuente (4).

Estudios sugieren que los estilos de vida pueden impactar en el riesgo de enfermar y morir de Covid 19. Se plantea que la sintomatología del enfermo de Covid-19 puede ser una interacción de variables biológicas y estilos de vida como pueden ser la edad, las comorbilidades, hábito de fumar, actividad física entre otros (5). También se ha documentado la asociación de variables sociales como el desarrollo social, PIB, estado de salud de la población y COVID-19 cuando coexiste con enfermedades crónicas no transmisibles como diabetes mellitus, hipertensión arterial, insuficiencia renal crónica y obesidad (6).

La OMS y los gobiernos locales ha intentado disminuir la pandemia y sus efectos letales; sin embargo, los reportes científicos 
son heterogéneos y no concluyentes en identificar qué factores sociodemográficos pudiesen estar asociados a la infección y mortalidad por Covid-19. Por ello, el objetivo de la investigación fue asociar factores sociodemográficos y de salud de la población con la infección y muerte por COVID 19.

\section{MATERIALES Y MÉTODOS}

Se empleó un diseño de análisis ecológico de tipo longitudinal. Las variables dependientes fueron Infectados $/ 10^{6}$ habitantes y Muertes $/ 10^{6}$ habitantes. El análisis consideró 11 variables independientes: \% población en inactividad física, \% población con 65 años de edad o más, PIB per cápita, \% pobreza extrema, tasa de mortalidad cardiovascular, prevalencia de diabetes, mujeres fumadoras, \% fumadores masculinos, instalaciones para lavarse las manos, camas de hospital $/ 10^{3}$ habitantes, esperanza de vida, índice de desarrollo humano.

Los datos fueron obtenidos de la organización Our World in Data con el patrocinio de la Universidad de Oxford, que son de acceso completamente abierto bajo la licencia Creative Commons. Esta organización autoriza a cualquier investigador a usar, distribuir y reproducir la información sobre el Covid 19 en cualquier medio. Los datos mostraron la información oficial de cada país como fuente original documentada, desde que se reportó el primer caso de la pandemia hasta la última actualización realizada en el mes de junio del año 2021 considerando cada día reportado como un corte transversal.

Se estudiaron tres momentos desde que inició la pandemia: hasta el 30 de junio del año 2020, hasta el 30 de noviembre del año 2020 y hasta el 4 de junio del año 2021. Todos los momentos corresponden a picos de infección conocidos como olas de infección (7). La población estuvo conformada por 219 países pertenecientes a los cinco continentes. La muestra se conformó por 91 países que presentaron datos completos en los 3 cortes temporales estudiados.

Losresultadossepresentanendossecciones. La primera presentó las correlaciones entre variables. Se utilizó un modelo de correlación de Pearson. Las correlaciones por debajo de 0,20 se consideraron muy bajas, las superiores a este valor y por debajo de 0,40. se consideran bajas. Las correlaciones bilaterales fueron significativas en el nivel $0,010\left(^{* \star}\right)$ y $0,050\left(^{(}\right)$. La segunda sección fue inferencial y presentó los valores predictivos de los dos modelos. Se utilizó un modelo de regresión lineal múltiple ( $R^{2}$ ajustado) obtenido mediante el software SPSS 25. Se desarrolló un modelo para la variable Infectados $/ 10^{6}$ habitantes y otro para la variable Muertes $/ 10^{6}$ habitantes. Se utilizó el método escalonado para elegir las variables que mejor explican el modelo $(p<0,050)$. Se interpretaron los resultados mediante los coeficientes no estandarizados según cada variable explicativa. 


\section{RESULTADOS}

La Tabla 1 muestra la correlación existente entre las variables independientes e Infectados - Muerte por Covid-19 (Tabla 1). Por otro lado, se generó el modelo de regresión lineal multivariado para las variables Infectados y Muertes por Covid -19 (Tablas 2 y 3) para cada corte temporal.

Se encontró correlaciones significativas positivas y negativas entre la mayoría de las variables independientes y dependientes para los diferentes momentos estudiados (Tabla 1).

Un total de siete variables presentaron correlación positivas débiles y moderadas con
Infectados y Muerte por Covid-19: porcentaje de personas con edad igual o superior a los 65 años, el PIB per cápita, el porcentaje de mujeres fumadoras, la presencia de instalaciones para lavarse las manos, la cantidad de camas de hospital por mil habitantes, la esperanza de vida, así como el Índice de Desarrollo Humano (IDH). Se observaron correlaciones negativas significativas de baja fortaleza para Infectados y Muertes con la pobreza extrema de los países y la tasa de mortalidad cardiovascular. No se encontró correlación significativa de Infectados o Muertes con los fumadores masculinos y prevalencia de diabetes.

Tabla 1. Coeficientes de correlación de Pearson de las variables independientes con respecto a Infecciones totales por millón de habitantes y Muertes totales por millón de habitantes debido a Covid-19. $\mathrm{N}=180$.

\begin{tabular}{lcccccc}
\hline & \multicolumn{3}{c}{ Casos totales por millón } & \multicolumn{3}{c}{ Muertes totales por millón } \\
& Jun. 2020 & Nov. 2020 & Jun. 2021 & Jun. 2020 & Nov. 2020 & Jun. 2021 \\
\hline \% población mayor a 64 años & 0,07 &, $481^{\star \star}$ &, $563^{\star \star}$ &, $419^{\star \star}$ &, $470^{\star \star}$ &, $544^{\star \star}$ \\
PIB per cápita &, $595^{\star \star}$ &, $522^{\star \star}$ &, $473^{\star \star}$ &, $360^{\star \star}$ &, $268^{\star \star}$ &, $229^{\star \star}$ \\
Pobreza extrema &,$- 319^{\star \star}$ &,$- 464^{\star \star}$ &,$- 512^{\star \star}$ &,$- 275^{\star \star}$ &,$- 437^{\star \star}$ &,$- 425^{\star \star}$ \\
Tasa de mortalidad cardiovascular &,$- 264^{\star \star}$ &,$- 285^{\star \star}$ &,$- 292^{\star \star}$ &,$- 368^{\star \star}$ &,$- 277^{\star \star}$ &,$- 204^{\star \star}$ \\
Prevalencia de diabetes &, $191^{\star}$ & 0,047 & 0,017 & $-0,086$ & 0,006 & $-0,007$ \\
Mujeres fumadoras & 0,09 &, $530^{\star \star}$ &, $593^{\star \star}$ &, $388^{\star \star}$ &, $496^{\star \star}$ &, $580^{\star \star}$ \\
Hombres fumadores & $-0,056$ & 0,055 & 0,104 & $-0,091$ & $-0,004$ & 0,086 \\
Instalaciones para lavarse las manos &, $363^{\star \star}$ &, $532^{\star \star}$ &, $548^{\star \star}$ &, $348^{\star \star}$ &, $465^{\star \star}$ &, $475^{\star \star}$ \\
Camas de hospital por mil & 0,026 &, $265^{\star \star}$ &, $333^{\star \star}$ & 0,102 &, $184^{\star}$ &, $289^{\star \star}$ \\
Esperanza de vida &, $347^{\star \star}$ &, $541^{\star \star}$ &, $578^{\star \star}$ &, $391^{\star \star}$ &, $473^{\star \star}$ &, $464^{\star \star}$ \\
Índice de desarrollo humano &, $345^{\star \star}$ &, $566^{\star \star}$ &, $618^{\star \star}$ &, $396^{\star \star}$ &, $486^{\star \star}$ &, $490^{\star \star}$ \\
\hline
\end{tabular}

**. La correlación es significativa en el nivel 0,01 (bilateral).

*. La correlación es significativa en el nivel 0,05 (bilateral).

Fuente: Our World in Data. 
El análisis temporal de las correlaciones entre Infectados $/ 10^{6}$ habitantes mostraron una tendencia al aumento de la fortaleza de asociación para todas las variables independientes con excepción del PIB, la cual tuvo una disminución de la asociación. Similar comportamiento fue encontrado para las correlaciones entre Muertes $/ 10^{6}$ habitantes y las variables independientes estudiadas.

\section{Modelos de regresión lineal múltiple para Infectados y muertes por Covid-19}

Los resultados de la modelación mediante Regresión lineal múltiple para las variables Infectados $/ 10^{6}$ habitantes y Muertes $/ 10^{6}$ habitantes para los tres cortes temporales se muestran en las Tablas 2 y 3 respectivamente.
El número de Infectados $/ 10^{6}$ habitantes es modelado exclusivamente por la variable independiente porcentaje de la población con edad igual o mayor a 65 años en los tres cortes temporales realizados. El resto de las variables fueron excluidas por ser no significativas. El poder explicativo del modelo se fue incrementando para los diferentes momentos desde junio 2020, noviembre 2020 y junio 2021 al apreciarse un aumento del coeficiente de determinación $\left(R^{2}\right)$ de 0,263 hasta 0,563 . El efecto de la variable independiente sugiere que por cada incremento del $1 \%$ en la proporción de la población con edad igual o mayor a 65 años se producen 5.269 nuevos Infectados $/ 10^{6}$ habitantes.

Tabla 2. Modelo de regresión lineal para infectados por cada millón de habitantes. $\mathrm{n}=91$ países.

\begin{tabular}{|c|c|c|c|c|c|c|c|c|c|}
\hline \multirow{2}{*}{ Fecha } & \multirow{2}{*}{ Modelo } & & \multicolumn{2}{|c|}{$\begin{array}{l}\text { Coeficientes no } \\
\text { estandarizados }\end{array}$} & \multirow{2}{*}{$\begin{array}{c}\text { Coeficientes } \\
\text { estandarizados } \\
\text { Beta }\end{array}$} & \multirow{2}{*}{$\mathbf{t}$} & \multirow{2}{*}{ Sig. } & \multicolumn{2}{|c|}{$\begin{array}{l}95 \% \text { intervalo de } \\
\text { confianza para B }\end{array}$} \\
\hline & & & B & $\begin{array}{l}\text { Desv. } \\
\text { Error }\end{array}$ & & & & $\begin{array}{l}\text { Límite } \\
\text { inferior }\end{array}$ & $\begin{array}{l}\text { Límite } \\
\text { superior }\end{array}$ \\
\hline \multirow[t]{2}{*}{ Jun. 2020} & $R^{2}=0,263$ & Constante & $-629,022$ & 405,505 & & $-1,551$ & ,128 & $-1446,801$ & 188,757 \\
\hline & & 65 años más & 268,261 & 65,666 & ,529 & 4,085 & ,000 & 135,832 & 400,690 \\
\hline \multirow[t]{2}{*}{ Nov. 2020} & $R^{2}=0,531$ & (Constante) & $-6818,305$ & 2095,297 & & $-3,254$ & ,002 & $-11043,874$ & $-2592,736$ \\
\hline & & 65 años más & 2419,630 & 339,307 & ,736 & 7,131 & ,000 & 1735,353 & 3103,907 \\
\hline \multirow[t]{2}{*}{ Jun. 2021} & $R^{2}=0,533$ & (Constante) & $-13114,092$ & 4544,625 & & $-2,886$ & ,006 & $-22279,201$ & $-3948,983$ \\
\hline & & 65 años más & 5269,180 & 735,944 & ,737 & 7,160 & ,000 & 3785,008 & 6753,352 \\
\hline
\end{tabular}

a. Variable dependiente: V. Dependiente 1 = Casos totales por millón.

Fuente: Our World in Data. 
La Tabla 3 expone los resultados de los modelos de regresión lineal que explican las muertes/106 habitantes en las tres fechas estudiadas. En junio del 2020 se observó que la única variable incluida con significación estadística fue el IDH, con un poder explicativo bajo R2 (ajustado)=0,212. Sin embargo, en noviembre del 2020, la variable explicativa del modelo fue la fracción de la población con 65 o más años con R2 (ajustado)=0,470, mientras que en junio del año 2021 se advierten dos variables explicativas en el modelo: la fracción de la población con 65 o más años y el porcentaje de mujeres fumadoras del país, R2 (ajustado) $=0,598$. Según este último modelo, por cada punto porcentual en la edad mayor a los 65 años se incrementa 131 muertes por Covid-19, así como, por cada punto porcentual en el número de mujeres fumadoras, se aumenta 35 muertes por Covid 19.

Tabla 3. Modelo de regresión lineal para la cantidad de muertos por cada millón de habitantes.

\begin{tabular}{|c|c|c|c|c|c|c|c|c|c|}
\hline \multirow{2}{*}{ Fecha } & \multirow{2}{*}{ Modelo } & & \multicolumn{2}{|c|}{$\begin{array}{l}\text { Coeficientes no } \\
\text { estandarizados }\end{array}$} & \multirow{2}{*}{$\begin{array}{c}\text { Coeficientes } \\
\text { estandarizados } \\
\text { Beta }\end{array}$} & \multirow{2}{*}{$\mathbf{t}$} & \multirow{2}{*}{ Sig. } & \multicolumn{2}{|c|}{$\begin{array}{l}95 \% \text { intervalo de } \\
\text { confianza para B }\end{array}$} \\
\hline & & & B & $\begin{array}{l}\text { Desv. } \\
\text { Error }\end{array}$ & & & & $\begin{array}{l}\text { Límite } \\
\text { inferior }\end{array}$ & $\begin{array}{l}\text { Límite } \\
\text { superior }\end{array}$ \\
\hline \multirow[t]{2}{*}{ Jun. 2020} & $R^{2}=0,212$ & Constante & $-116,342$ & 43,956 & & $-2,647$ & ,012 & $-205,326$ & $-27,358$ \\
\hline & & $\begin{array}{l}\text { Índice de } \\
\text { desarrollo } \\
\text { humano }\end{array}$ & 232,141 & 68,393 & ,482 & 3,394 & ,002 & 93,686 & 370,596 \\
\hline \multirow[t]{2}{*}{ Nov. 2020} & $R^{2}=0,483$ & (Constante) & $-149,794$ & 59,370 & & $-2,523$ & ,016 & $-269,784$ & $-29,804$ \\
\hline & & 65 años más & 57,521 & 9,413 & ,695 & 6,111 & ,000 & 38,497 & 76,545 \\
\hline \multirow[t]{3}{*}{ Jun. 2021} & $R^{2}=0,598$ & (Constante) & $-455,918$ & 127,587 & & $-3,573$ & ,001 & $-713,399$ & $-198,438$ \\
\hline & & 65 años más & 131,484 & 24,656 & 610 & 5,333 & ,000 & 81,726 & 181,241 \\
\hline & & $\begin{array}{l}\text { Mujeres } \\
\text { fumadoras }\end{array}$ & 35,502 & 15,557 & 261 & 2,282 &, 028 & 4,108 & 66,897 \\
\hline
\end{tabular}

a. Variable dependiente: V. Dependiente 1= Casos totales por millón.

Fuente: Our World in Data.

\section{Discusión}

El comportamiento de la salud de las poblaciones se define por un conjunto de factores determinantes de salud de variada naturaleza. Existen diversas investigaciones que han intentado establecer los principales factores asociados a la Infección y Muerte por Covid-19. Los resultados aún no son concluyentes por existir divergencia y discrepancias en los hallazgos científicos.

Las fortalezas y signo positivo de las correlaciones de variables indicadoras de desarrollo con la Infección y Muerte por Covid-19 sugieren que el epicentro de la pandemia ha estado alrededor de los países con indicadores de alto nivel de desarrollo. 
Altos niveles de PIB o de IDH deberían tener una correlación negativa con las infecciones y muertes por enfermedades trasmisibles.

Las evidencias empíricas sobre la relación entre el IDH y la infección y muerte por Covid 19 es escasa. Un estudio realizado en Italia coincide con los hallazgos, demostrando la asociación con la tasa de infección y la tasa de letalidad de COVID-19 (2).

Estudios previos han encontrado una correlación entre el IDH con eventos de salud relacionados a enfermedades crónicas trasmisibles y no trasmisibles como el cáncer. El metaanálisis y estudio ecológico referido evidenció la correlación negativa entre diferentes tipos de cáncer y el PIB, mientras que el cáncer de mama y ovario lo hicieron de manera positiva. El valor explicativo del IDH ha sido significativo para enfermedades como tuberculosis, malaria y suicidio; sin embargo no ha sido relevante en explicar las diarreas infecciosas, las enfermedades cardiovasculares y la infección por leishmaniasis (8).

Al ser el IDH un indicador multidimensional que integra la educación, la esperanza de vida y el PIB/persona, su valor como marcador de salud es inespecífico. Esto sugiere que el IDH es una variable a considerar según la enfermedad, sus particularidades y los determinantes propios que orientan el progreso de la enfermedad en la población.

Similar a los resultados encontrados, otras investigaciones a nivel local asocian el PIB con la infección y muerte por Covid-19. En Italia se encontró correlación positiva en un estudio de 21 regiones y provincias italianas (9). Esto reafirma a los países desarrollados como el epicentro de la pandemia a nivel mundial.

También pudiese parecer contradictorio que indicadores de la infraestructura en salud como el número de camas $/ 10^{3}$ habitantes y sitios donde lavarse las manos se asocien a la infección y la muerte. Estudios encontraron asociación inversa entre estas variables en momentos alejados de los picos de infección y muerte a nivel mundial, lo cual es opuesto a la investigación realizada y puede explicar la diferencia de resultados. Sin embargo, si el análisis se realiza en los momentos picos de infectados o muertes la correlación se debilita y la razón de momios disminuye a un valor cercano a 1 (10).

\section{¿Cómo explicar esta aparente paradoja que asocia positivamente el desarrollo y la infraestructura en salud con el aumento de la infección y muerte por Covid-19?}

La paradoja puede ser explicada desde la novedad de la infección y la falta de preparación de los sistemas sanitarios y de salud. Los sistemas de salud de todo el planeta fueron sorprendidos por el virus. Existió un total desconocimiento sobre el agente etiológico y sus vías de contagio. Tampoco existían conocimientos sobre la patogénesis y su posible tratamiento. El diagnóstico fue incierto en un inicio y a posteriori la diversidad de métodos y su interpretación diversa provocó discrepancias en cuanto al uso clínico y epidemiológico. Aún persiste la ausencia de fármacos específicos contra el Covid-19. El volumen de trabajo en las UCI 
fue desbordante con respecto a la demanda de camas y respiradores. No existían políticas ni infraestructura en salud que intervinieran la epidemia por el SARS-CoV-2. Tampoco existían vacunas preventivas o curativas contra el virus. Todo ello hizo que, aunque los países desarrollados tuviesen indicadores de salud óptimos, estos no lograron impactar en el diagnóstico, tratamiento y prevención de la infección y muerte por Covid-19. Es por ello, que las búsquedas de otros determinantes de salud pueden ser más relevantes para explicar el comportamiento de la pandemia a nivel global.

La identificación de determinantes de salud como poblaciones susceptibles, grupos etáreos más afectados y variables biomédicas como enfermedades crónicas no trasmisibles también han sido objeto de estudio. Investigaciones de cohortes y metaanálisis en población de diferentes países han confirmado a los adultos mayores como un factor de riesgo para la infección y muerte por Covid-19 (3). Estudios durante la pandemia señalaron que el $90 \%$ de casos mortales fueron de personas mayores a 50 años, sugiriendo que a medida que aumenta la edad de los adultos mayores también aumenta la letalidad de la enfermedad (11). Sin embargo, estudios ecológicos a nivel local o regional han detectado que la población joven incrementa el número de infectados, contradiciendo los hallazgos anteriores (12).

La presencia de factores de salud predisponentes como enfermedades cónicas ha sido ampliamente explorada por diversas investigaciones. Enfermedades como diabetes
(13), hipertensión arterial, obesidad (14), insuficiencia renal (15) han sido asociadas a una mayor infección y mortalidad ante Covid-19 en estudios empíricos con diferentes poblaciones. Las estimaciones de China muestran que la mortalidad sin comorbilidades fue del $0,9 \%$, lo que aumentó a $10,5 \%$ con enfermedad cardiovascular, $6,3 \%$ con enfermedad pulmonar obstructiva crónica, $6 \%$ con hipertensión arterial y $5,6 \%$ con cáncer (16).

Estudiosecológicosrealizadosanivelglobal en 185 países confirman la asociación positiva entre enfermedades crónicas no transmisibles y la infección y muerte por Covid-19 (17). Otras investigaciones contradicen los hallazgos anteriores. En Irán no se encontró que las comorbilidades como diabetes, obesidad y asma explicaran la evolución clínica tórpida del Covid-19 (14).

Investigaciones en China e Italia han demostrado un curso más severo de la infección por SARS-CoV-2 en pacientes diabéticos, que requiere traslado a la unidad de cuidados intensivos $(\mathrm{UCl})$ y ventilación mecánica. Un estudio de una serie de casos en el área de Nueva York mostró una incidencia de diabetes de aproximadamente el $34 \%$ entre 5.700 pacientes hospitalizados con COVID-19 (18). Las observaciones realizadas con pacientes con COVID-19 en todo el mundo deberían llevar a la probable conclusión de que los pacientes con diabetes como enfermedad comórbida tienen un mayor riesgo de experimentar efectos secundarios graves a causa de una infección, sin embargo, ello no se puede corroborar con los resultados del presente estudio. 
Algunos autores han propuesto que, aunque la diabetes mellitus provoca cambios en la expresión de ACE-2 y DPP-4, su repercusión no es relevante en el contagio y la muerte (19). El mecanismo propuesto, de manera similar a lo que ocurre con otras enfermedades infecciosas, plantea que los pacientes diabéticos tienen un sistema inmune debilitado lo que favorece la infección de virus y microorganismos. Esta situación repercute en el curso de la enfermedad incrementando la severidad clínica y la mortalidad.

Las enfermedades cardiovasculares asociadasalCovid-19incluyenlesiónmiocárdica aguda, miocarditis, síndromes coronarios agudos, arritmias y tromboembolismo venoso (20). Por otro lado, las terapias utilizadas para tratar la enfermedad COVID-19 pueden tener interacciones farmacológicas, complicando el pronóstico de los pacientes (21). La ausencia de correlación y poder explicativo en ciertas investigaciones puede relacionarse con el momento del estudio, donde el pico de muerte e infección relacione otras variables con mayor poder explicativo (22).

El efecto del tabaquismo en los enfermos de Covid-19 ha sido documentado como un factor de riesgo de infección y muerte. Revisiones sobre la literatura han sistematizado el incremento del riesgo de infección y muerte por Covid-19 en modelos in vivo, in vitro $y$ epidemiológicos con seres humanos (23). Un estudio desarrollado en España sugiere que la reducción del consumo de tabaco se asocia a una reducción del riesgo de la mortalidad (24). El acto de fumar implica actividades riesgosas que pueden promover la difusión viral como son toser, estornudar, llevarse las manos a la boca, intercambio de sustancias con superficies y aerosoles. Los autores también plantean que el humo del tabaco provoca daño celular y tisular del epitelio pulmonar por acción viral, vinculándolo a la disminución de la respuesta inmune innata en los tejidos expuestos al humo del tabaco. Se ha encontrado también un incremento del uso del tabaco en poblaciones de diversos países como China, Inglaterra, Italia y Estados Unidos durante la pandemia, lo que pudiese enmascarar el efecto de esta variable. Sin embargo, otras investigaciones no han podido comprobar que existe riesgo de infección o muerte por el hábito de fumar (25) o reportan mayor hospitalización en pacientes exfumadores que fumadores actuales (26).

La influencia de fumar sobre la infección y mortalidad según el sexo también arroja resultados diferentes a la presente investigación. Resultados en cohortes del norte de África han encontrado un incremento del riesgo de muerte y el tiempo de estancia en $\mathrm{UCl}$ en pacientes masculinos fumadores enfermos de Covid-19. El sexo femenino no mostró cambios en el OR calculado (27). Se propone que mecanismos hormonales como la secreción de estrógenos y testosterona puedan estar relacionados a un peor pronóstico en el sexo masculino.

¿Cuáles fueron las causas que desencadenaron que la pandemia haya tenido su epicentro en los países desarrollados? Quizás profundizar en variables relacionadas con la movilidad humana (migración y turismo), 
la demografía como la densidad poblacional y variantes étnicas pudiese ser la respuesta. Investigaciones ecológicas ajustadas al turismo por países (17) y la densidad poblacional (28) han encontrado una correlación positiva con las muertes por Covid 19, lo que reafirma la idea planteada.

La búsqueda de modelos que reflejen los determinantes no muestra unicidad de criterios, donde el efecto de múltiples variables es coherente con un fenómeno complejo. Estudios realizados a nivel global han identificado variables como la vacunación con BCG y los viajeros que arriban al país (4), baja densidad poblacional, población con mayor proporción de sexo femenino (29) lo que contradice hallazgos de otras investigaciones.

Las contradicciones y heterogeneidad de los determinantes alrededor de la infección o muerte por Covid-19 refuerzan la concepción de la integralidad de la atención en salud, donde existen un conjunto de determinantes en el orden biológico, social, cultural y político que se integran de manera compleja en la salud de la población. Lograr un equilibrio entre los determinantes por acción coordinada de los gobiernos y las políticas en salud, permitirá potenciar las fortalezas y minimizar las debilidades en aras de incrementar la salud y el bienestar de las poblaciones en el marco de la pandemia de Covid-19

\section{Limitaciones}

La matriz de Our World In Data aporta datos de más de 200 países, sin aportar los valores individuales relacionados a cada contagio y muerte para establecer los factores de riesgo. La presencia de variables confusoras o mediadoras del efecto son de difícil control. En tal sentido, los hallazgos, se limitan a exponer los resultados ecológicos reportados por las cantidades de infectados y fallecidos a partir del primer caso hasta la última actualización realizada en junio del año 2021. De este modo, las unidades de análisis constituyen cada país que ha reportado datos en estas fechas y los resultados no pueden ser extrapolados a casos individuales.

\section{CONCLUSIONES}

La existencia de determinantes de salud de orden biológico, social, cultural y político impactan de manera diversa sobre la infección y muerte por Covid-19. La compleja relación entre estas y el impacto temporal sobre el fenómeno estudiado hacen que su generalización sea científicamente no adecuada. Las similitudes y contradicciones con otras investigaciones reafirman el carácter complejo de la infección y muerte por Covid 19 dentro de las ciencias de la salud replanteando la jerarquía de los determinantes en salud en época de pandemia.

\section{REFERENCIAS BIBLIOGRÁFICAS}

1. Wang $Y$, Wang $Y$, Chen $Y$, Qin $Q$. Unique epidemiological and clinical features of the emerging 2019 novel coronavirus pneumonia (COVID-19) implicate special control measures. Journal of Medical Virology [Internet]. 2020 [citado 21 de septiembre de 2021];92(6):568- 
76. Disponible en: https://onlinelibrary.wiley. com/doi/abs/10.1002/jmv.25748

2. Liu Z, Magal P, Webb G. Predicting the number of reported and unreported cases for the COVID-19 epidemics in China, South Korea, Italy, France, Germany and United Kingdom. Journal of Theoretical Biology [Internet]. 21 de enero de 2021 [citado 21 de septiembre de 2021];509:110501. Disponible en: https:// www.sciencedirect.com/science/article/pii/ S0022519320303568

3. Parohan $M$, Yaghoubi S, Seraji A, Javanbakht $\mathrm{MH}$, Sarraf P, Djalali M. Risk factors for mortality in patients with Coronavirus disease 2019 (COVID-19) infection: a systematic review and meta-analysis of observational studies. The Aging Male [Internet]. 4 de diciembre de 2020 [citado 1 de septiembre de 2021];23(5):141624. Disponible en: https://doi.org/10.1080/136 85538.2020.1774748

4. Pana TA, Bhattacharya S, Gamble DT, Pasdar Z, Szlachetka WA, Perdomo-Lampignano $\mathrm{JA}$, et al. Country-level determinants of the severity of the first global wave of the COVID-19 pandemic: an ecological study. BMJ Open [Internet]. 1 de febrero de 2021 [citado 19 de septiembre de 2021];11(2):e042034. Disponible en: https://bmjopen.bmj.com/content/11/2/ e042034

5. Rozenfeld $Y$, Beam J, Maier $H$, Haggerson W, Boudreau K, Carlson J, et al. A model of disparities: risk factors associated with COVID-19 infection. International Journal for Equity in Health [Internet]. 29 de julio de 2020 [citado 1 de septiembre de 2021];19(1):126. Disponible en: https://doi.org/10.1186/s12939020-01242-z

6. Izcovich A, Ragusa MA, Tortosa F, Marzio MAL, Agnoletti C, Bengolea A, et al. Prognostic factors for severity and mortality in patients infected with COVID-19: A systematic review. PLOS ONE [Internet]. 17 de noviembre de 2020 [citado 1 de septiembre de 2021];15(11):e0241955. Disponible en: https:// journals.plos.org/plosone/article?id=10.1371/ journal.pone.0241955

7. González-Castro A, Cuenca Fito E, Fernandez A, Escudero Acha $P$, Rodríguez Borregán JC, Peñasco Y. Primera y segunda oleada de enfermedad por coronavirus-19: un estudio comparativo en pacientes hospitalizados en una $\mathrm{UCl}$ de un hospital universitario de tercer nivel. Med Intensiva [Internet]. [citado 21 de septiembre de 2021]; Disponible en: http://www.medintensiva.org/ es-primera-segunda-oleada-enfermedad-poravance-S0210569121000292

8. Higuita-Gutiérrez LF, Cardona-Arias JA, Higuita-Gutiérrez LF, Cardona-Arias JA. Índice de desarrollo humano y eventos de salud pública: revisión sistemática de la literatura 1990-2015. Revista Facultad Nacional de Salud Pública [Internet]. abril de 2018 [citado 17 de septiembre de 2021];36(1):5-16. Disponible en: http://www.scielo.org.co/scielo. php?script=sci_abstract\&pid $=$ S0120-386X2018 $000100005 \& \operatorname{lng}=$ en\&nrm=iso\&tlng=es

9. Lippi G, Henry BM, Mattiuzzi C, Bovo C. The death rate for COVID-19 is positively associated with gross domestic products: Coronavirus and gross domestic product. Acta Bio Medica Atenei Parmensis [Internet]. 11 de mayo de 2020 [citado 18 de septiembre de 2021];91(2):224-5. Disponible en: https://doi.org/10.23750/abm. v91i2.9514

10. Arsang-Jang S, Belasi MT, Najafi F, Darbandi $M$, Raza MZ, Akhuanzada $H$, et al. The association between healthcare resources, non-communicable diseases, and Covid-19 mortality: an epidemiological study of 139 countries [Internet]. In Review; 2021 abr [citado 17 de septiembre de 2021]. Disponible en: https://www.researchsquare.com/article/ rs-379944/v1

11. Pijls BG, Jolani S, Atherley A, Derckx RT, Dijkstra JIR, Franssen GHL, et al. Demographic risk factors for COVID-19 infection, severity, ICU admission and death: a meta-analysis 
of 59 studies. BMJ Open [Internet]. 1 de enero de 2021 [citado 1 de septiembre de 2021];11(1):e044640. Disponible en: https:// bmjopen.bmj.com/content/11/1/e044640

12. Whittle RS, Diaz-Artiles A. An ecological study of socioeconomic predictors in detection of COVID-19 cases across neighborhoods in New York City. BMC Medicine [Internet]. 4 de septiembre de 2020 [citado 21 de septiembre de 2021];18(1):271. Disponible en: https://doi. org/10.1186/s12916-020-01731-6

13. Pérez-Martínez $P$, Carrasco Sánchez FJ, Carretero Gómez J, Gómez-Huelgas R. Resolviendo una de las piezas del puzle: COVID-19 y diabetes tipo 2. Revista Clínica Española [Internet]. 1 de noviembre de 2020 [citado 18 de septiembre de 2021];220(8):50710. Disponible en: https://www.sciencedirect. com/science/article/pii/S0014256520301351

14. Khorrami Z, Nili S, Sharifi H, Eybpoosh S, Shokoohi M. Association of cigarette smoking, obesity, and underlying medical conditions with COVID-19 hospitalization and mortality in Iran: A nationwide retrospective ecological study. Med J Islam Repub Iran [Internet]. 8 de octubre de 2020 [citado 18 de septiembre de 2021];34:133. Disponible en: https://www.ncbi. nlm.nih.gov/pmc/articles/PMC7787023/

15. Bhargava A, Fukushima EA, Levine $M$, Zhao W, Tanveer F, Szpunar SM, et al. Predictors for severe Covid-19 infection. Clinical Infectious Diseases [Internet]. 5 de noviembre de 2020 [citado 1 de septiembre de 2021];71(8):19628. Disponible en: https://doi.org/10.1093/cid/ ciaa674

16. Yang J, Zheng Y, Gou X, Pu K, Chen Z, Guo $Q$, et al. Prevalence of comorbidities and its effects in patients infected with SARS-CoV-2: a systematic review and meta-analysis. Int J Infect Dis. mayo de 2020;94:91-5.

17. Azarpazhooh MR, Morovatdar N, Avan A, Phan TG, Divani AA, Yassi N, et al. Covid-19 pandemic and burden of noncommunicable diseases: an ecological study on data of 185 countries. Journal of Stroke and Cerebrovascular Diseases [Internet]. 1 de septiembre de 2020 [citado 16 de septiembre de 2021];29(9):105089. Disponible en: https:// www.sciencedirect.com/science/article/pii/ S1052305720305073

18. Gasmi A, Peana M, Pivina L, Srinath S, Gasmi Benahmed A, Semenova Y, et al. Interrelations between COVID-19 and other disorders. Clin Immunol [Internet]. marzo de 2021 [citado 21 de septiembre de 2021];224:108651. Disponible en: https://www.ncbi.nlm.nih.gov/ pmc/articles/PMC7833539/

19. Peric S, Stulnig TM. Diabetes and COVID-19. Wien Klin Wochenschr [Internet]. 1 de julio de 2020 [citado 18 de septiembre de 2021];132(13):356-61. Disponible en: https:// doi.org/10.1007/s00508-020-01672-3

20. Clerkin KJ, Fried JA, Raikhelkar J, Sayer G, Griffin JM, Masoumi A, et al. Covid-19 and cardiovascular disease. Circulation [Internet]. 19 de mayo de 2020 [citado 19 de septiembre de 2021];141(20):1648-55. Disponible en: https://www.ahajournals.org/doi/full/10.1161/ CIRCULATIONAHA.120.046941

21. Alfonso Rodriguez E, Llerena Rojas LD, Rodríguez Nande LM, Alfonso Rodriguez E, Llerena Rojas LD, Rodríguez Nande LM. Consideraciones para pacientes con enfermedades cardiovasculares durante la pandemia de la COVID-19. Revista Cubana de Investigaciones Biomédicas [Internet]. septiembre de 2020 [citado 5 de junio de 2021];39(3). Disponible en: http://scielo.sld. cu/scielo.php?script=sci_abstract\&pid=S0864$03002020000300023 \& \operatorname{lng}=$ es\&nrm=iso\&tlng= es

22. DuPre NC, Karimi S, Zhang $\mathrm{CH}$, Blair L, Gupta A, Alharbi LMA, et al. County-level demographic, social, economic, and lifestyle correlates of COVID-19 infection and death trajectories during the first wave of the pandemic in the United States. Science of The Total Environment [Internet]. 10 de septiembre de 2021 [citado 19 de septiembre de 2021];786:147495. Disponible en: https:// 
www.sciencedirect.com/science/article/pii/ S0048969721025663

23. Haddad C, Bou Malhab $S$, Sacre $H$, Salameh P. Smoking and COVID-19: A Scoping Review. Tob Use Insights [Internet]. 1 de enero de 2021 [citado 20 de septiembre de 2021];14:1179173X21994612. Disponible en: https://doi.org/10.1177/1179173X21994612

24. López-Bueno R, Calatayud J, Casaña J, Casajús JA, Smith L, Tully MA, et al. COVID-19 Confinement and Health Risk Behaviors in Spain. Front Psychol [Internet]. 2020 [citado 5 de junio de 2021];11. Disponible en: https:// www.frontiersin.org/articles/10.3389/ fpsyg.2020.01426/full

25. Padilla-Raygoza N, Vega-Jimenez OU, Juárez AG, Gallardo-Luna M de J, NavarroOlivos $E$, León-Verdin MG. Effect of comorbidities on mortality from Covid-19 in Mexico: an ecological study. Biomedical and Pharmacology Journal [Internet]. 30 de marzo de 2021 [citado 19 de septiembre de 2021];14(1):7-13. Disponible en: https:// biomedpharmajournal.org/vol14nol/effectof-co-morbidities-on-mortality-from-covid-19in-mexico-an-ecological-study/

26. Puebla Neira D, Watts A, Seashore J, Polychronopoulou E, Kuo Y-F, Sharma G. Smoking and risk of COVID-19 hospitalization. Respiratory Medicine [Internet]. 1 de junio de 2021 [citado 20 de septiembre de 2021];182:106414. Disponible en: https:// www.sciencedirect.com/science/article/pii/ S0954611121001207
27. Khalil A, Dhingra R, Al-Mulki J, Hassoun $M$, Alexis $N$. Questioning the sex-specific differences in the association of smoking on the survival rate of hospitalized COVID-19 patients. PLOS ONE [Internet]. 5 de agosto de 2021 [citado 20 de septiembre de 2021];16(8):e0255692. Disponible en: https:// journals.plos.org/plosone/article?id=10.1371/ journal.pone.0255692

28. Markovic S, Rodic A, Salom I, Milicevic O, Djordjevic M, Djordjevic M. COVID-19 severity determinants inferred through ecological and epidemiological modeling. arXiv:210900038 [q-bio] [Internet]. 31 de agosto de 2021 [citado 19 de septiembre de 2021]; Disponible en: http://arxiv.org/abs/2109.00038

29. Foo O, Hiu S, Teare D, Syed AA, Razvi S. A global country-level analysis of the relationship between obesity and COVID-19 cases and mortality. Diabetes, Obesity and Metabolism [Internet]. [citado 19 de septiembre de 2021];n/a(n/a). Disponible en: https:// onlinelibrary.wiley.com/doi/abs/10.1111/ dom.14523

Conflicto de intereses: Ninguno declarado por los autores. Financiación: Ninguna declarada por los autores.

Agradecimiento: Ninguno manifestado por los autores

\section{ACERCA DE LOS AUTORES}

Franz Chumbi Sigcho. Químico Farmaceuta de profesión. Maestrante en Diagnóstico Clínico y Molecular. Universidad Católica de Cuenca, Ecuador.

Carlos Román Collazo. PhD en Bioquímica, Biología Molecular, Bioética y Metodología de la Investigación. Director de proyectos de Investigación. Arbitro y editor de revistas científicas. Universidad Católica de Cuenca, Ecuador.
Juan Moreno Bravol. Médico general. Cursando el Primer año de Residencia en la especialización de pediatría en la Universidad del Azuay. Universidad Católica de Cuenca, Ecuador.

Jessica Velecela Chumbi. Médico General, Universidad de Cuenca. Cursado una especialización en Nefrología, Universidad Pontificia Católica de Rio de Janeiro. Médico residente de Hemodiálisis en Fresenius Medical Care. Universidad Católica de Cuenca, Ecuador. 